\title{
Artemisinin resistance without pfkelch13 mutations in Plasmodium falciparum isolates from Cambodia
}

\author{
Angana Mukherjee ${ }^{1 \dagger}$, Selina Bopp ${ }^{1 \dagger}$, Pamela Magistrado ${ }^{1}$, Wesley Wong ${ }^{1}$, Rachel Daniels ${ }^{1,2}$, Allison Demas ${ }^{1}$, \\ Stephen Schaffner ${ }^{2}$, Chanaki Amaratunga ${ }^{3}$, Pharath Lim³ ${ }^{3}$, Mehul Dhorda ${ }^{4,5}$, Olivo Miotto 6,7,8, \\ Charles Woodrow ${ }^{5,6}$, Elizabeth A. Ashley 5,6, Arjen M. Dondorp ${ }^{5,6,9}$, Nicholas J. White5, ${ }^{5,6}$, Dyann Wirth ${ }^{1,2}$, \\ Rick Fairhurst ${ }^{3}$ and Sarah K. Volkman 1,2,10*
}

\begin{abstract}
Background: Artemisinin resistance is associated with delayed parasite clearance half-life in vivo and correlates with ring-stage survival under dihydroartemisinin in vitro. Both phenotypes are associated with mutations in the PF3D7_1343700 pfkelch13 gene. Recent spread of artemisinin resistance and emerging piperaquine resistance in Southeast Asia show that artemisinin combination therapy, such as dihydroartemisinin-piperaquine, are losing clinical effectiveness, prompting investigation of drug resistance mechanisms and development of strategies to surmount emerging anti-malarial resistance.

Methods: Sixty-eight parasites isolates with in vivo clearance data were obtained from two Tracking Resistance to Artemisinin Collaboration study sites in Cambodia, culture-adapted, and genotyped for pfkelch 13 and other mutations including pfmdr 1 copy number; and the $\mathrm{RSA}_{0-3 \mathrm{~h}}$ survival rates and response to antimalarial drugs in vitro were measured for 36 of these isolates.
\end{abstract}

Results: Among these 36 parasites one isolate demonstrated increased ring-stage survival for a PfKelch13 mutation $\left(\mathrm{D} 584 \mathrm{~V}, \mathrm{RSA}_{0-3 \mathrm{~h}}=8 \%\right)$, previously associated with slow clearance but not yet tested in vitro. Several parasites exhibited increased ring-stage survival, yet lack pfkelch 13 mutations, and one isolate showed evidence for piperaquine resistance.

Conclusions: This study of 68 culture-adapted Plasmodium falciparum clinical isolates from Cambodia with known clearance values, associated the D584V PfKelch13 mutation with increased ring-stage survival and identified parasites that lack pfkelch13 mutations yet exhibit increased ring-stage survival. These data suggest mutations other than those found in pfkelch13 may be involved in conferring artemisinin resistance in P. falciparum. Piperaquine resistance was also detected among the same Cambodian samples, consistent with reports of emerging piperaquine resistance in the field. These culture-adapted parasites permit further investigation of mechanisms of both artemisinin and piperaquine resistance and development of strategies to prevent or overcome anti-malarial resistance.

Keywords: Plasmodium falciparum, Artemisinin resistance, pfkelch13, Piperaquine resistance

\footnotetext{
*Correspondence: svolkman@hsph.harvard.edu

${ }^{\dagger}$ Angana Mukherjee and Selina Bopp contributed equally to this work

1 Department of Immunology and Infectious Disease, Harvard T.H. Chan

School of Public Health, 665 Huntington Avenue, I-704, Boston, MA

02115, USA

Full list of author information is available at the end of the article
} 


\section{Background}

Artemisinin (ART) and related compounds provide the main class of anti-malarial drugs, and ART resistance in Plasmodium falciparum is one of the greatest threats to global efforts to control, eliminate and eradicate malaria. To forestall emergence and spread of ART resistance it was recommended that ART and its derivatives be used only in combination with a partner drug as an ART combination therapy (ACT), with over 400 million ACT treatments dispensed annually. It is conservatively estimated that 116,000 additional deaths would occur annually in the event of widespread ART resistance, with annual health costs of US $\$ 32$ million and productivity losses exceeding US\$385 million [1]. The magnitude of the health and economic threat posed by ART resistance serves as an urgent call to action to develop strategies that circumvent its spread [2]. Doing so requires understanding the underlying mechanisms of ART resistance.

Currently, ART resistance is prevalent across the Greater Mekong Sub-region (GMS) and centered on Cambodia, where it was first detected in 2007 [3, 4]. Concern for the spread of ART resistance outside of Southeast Asia led to the Tracking Resistance to Artemisinin Collaboration (TRAC) study that assessed and tracked ART resistance across 15 sites both in Asia and Africa [5]. Using parasite clearance as a measure of resistance, the TRAC project confirmed that ART-resistant $P$. falciparum was established in Cambodia, Laos, Myanmar, Thailand, and Vietnam.

Artemisinin resistance is indicated by either delays in parasite clearance from patients, or by increased in vitro parasite survival under dihydroartemisinin (DHA) in ring-stage survival assay $\left(\mathrm{RSA}_{0-3 \mathrm{~h}}\right)$ [6]. Sequencing parasites selected under increasing ART pressure identified pfkelch13 as a critical gene for conferring ART resistance [7]. Multiple mutations have been identified in this gene, and several key single nucleotide polymorphisms (SNPs) have been validated using a gene-editing approach $[8,9]$. However, the function of PfKelch13 and its role in ART resistance remain unclear.

Recent reports confirm that specific pfkelch13 mutations confer ring-stage survival [7, 8], and indicate possible mechanisms of ART resistance, including perturbation of haemoglobin processing [10-12], protein ubiquitination [13], increased expression of oxidative stress response [13, 14], unfolded protein response pathways [14], or phosphatidylinositide 3-kinase pathways [15]. While these results may all be relevant, a full understanding of the mechanisms involved in ART action and resistance has yet to emerge $[16,17]$.

One of the key partner drugs used with ART for malaria treatment in the GMS is piperaquine (PPQ), which has proved well tolerated and highly effective in areas where multi-drug-resistant $P$. falciparum is prevalent. However, emergence of PPQ resistance threatens to undermine this strategy in areas of increasing ART resistance $[18,19]$. Currently PPQ resistance is evident by higher PPQ half maximal effective concentration $\left(\mathrm{EC}_{50}\right)$ values and elevated recrudescence rates in settings where DHA-PPQ is in use and ART resistance is common $[18,19]$. Reported molecular markers of PPQ resistance include SNPs in pfcrt and de-amplification of a region on chromosome 5 that includes or is proximal to $p f m d r 1$ [20]. PPQ resistance is also associated with an amplification of plasmepsin $I I$ and $I I I$ [21, 22]. Yet, to date no mechanism has been established for PPQ resistance. Increasing resistance to both ART and PPQ in the same parasite population has motivated a call for use of triple combination therapy in certain malaria-endemic settings where ACT may have reduced efficacy $[18,23]$. Specifically, mefloquine (MFQ) has been suggested for combination with ART and PPQ given the inverse effects PPQ and MFQ pressure have on $p f m d r 1$ copy number variation $(\mathrm{CNV})$, as PPQ negatively selects $p f m d r 1 \mathrm{CNV}$, while MFQ positively selects $p f m d r 1 \mathrm{CNV}$ [18].

As part of the TRAC collaboration, 157 cryopreserved parasites were obtained from two sites in western Cambodia with a range of in vivo clearance phenotypes. Of these parasites, 68 were culture-adapted, and a sub-set of 36 parasites evaluated for their $\mathrm{RSA}_{0-3 \mathrm{~h}}$ phenotype. Using a high-resolution melt (HRM) genotyping assay for the most common pfkelch13 mutation (C580Y) and Sanger sequencing, pfkelch13 propeller mutations were tested for associations with both parasite in vivo clearance half-lives and in vitro parasite $\mathrm{RSA}_{0-3 \mathrm{~h}}$ survival rates. The analysis (1) identified parasites lacking pfkelch13 mutations, but exhibiting increased $\mathrm{RSA}_{0-3 \mathrm{~h}}$ survival phenotype (referred to as discordant parasites), suggesting that loci other than pfkelch13 may be involved in ART resistance; (2) demonstrated a new association between $\mathrm{D} 584 \mathrm{~V}$ and increased ring-stage survival; and, (3) detected a PPQ-resistant isolate among these parasites, consistent with other reports from this malariaendemic region. Although there is a strong association between pfkelch13 mutations and increased ring-stage survival, mutations in pfkelch13 are not necessary for this ART resistance phenotype. Furthermore, in vitro PPQ resistance is present among these culture-adapted Cambodian parasites, which will enable further investigation of PPQ resistance mechanisms.

\section{Methods}

These parasite samples were obtained with informed consent from patients enrolled in the TRAC study in the Pailin and Pursat sites located in Western Cambodia. Full details of this study, the approvals, and the clinical and 
laboratory methodologies have been reported in detail elsewhere [5].

\section{Culture-adaptation and maintenance of TRAC parasites} All parasite samples were collected under protocols approved by ethical review boards in Cambodia, at Oxford University and at the Harvard T. H. Chan School of Public Health. Culture-adaptation of parasites was accomplished by thawing cryopreserved material containing infected red blood cells (iRBCs) that had been mixed with glycerolyte. Parasites were maintained in fresh human blood $(\mathrm{O}+)$ and Hepes buffered RPMI media containing $12.5 \% \mathrm{AB}+$ human serum (heat inactivated and pooled). Cultures were placed in modular incubators and gassed with $1 \% \mathrm{O}_{2} / 5 \% \mathrm{CO}_{2} /$ balance $\mathrm{N}_{2}$ gas and incubated with rotation $(50 \mathrm{rpm})$ in a $37^{\circ} \mathrm{C}$ incubator (Additional file 1).

\section{Sample extraction}

Genetic material were extracted from filter papers (Whatman) and culture-adapted material using Promega DNA IQ Casework Pro Kit for Maxwell 16 (Promega Corp., Madison, WI, USA) and Qiagen (QIAmp DNA Blood Mini Kit) commercial kits, respectively, according to manufacturer instructions.

\section{Genotyping}

Development of a high-resolution melt (HRM) assay to screen populations for mutations around amino acid position 580 in the pfkelch13 locus. The forward primer (5'-GGCACCTTTGAATACCC-3'), reverse primer (5'-CATTAGTTCCACCAATGACA-3'), and unlabeled, blocked probe (5'-AGCTATGTGTATTGCTTTTGATblock-3') were amplified asymmetrically at $0.5,0.1$, and $0.4 \mu \mathrm{M}$, respectively with $1 \mathrm{ng}$ template DNA. After an initial 2 -min hold at $95{ }^{\circ} \mathrm{C}, 5$ or $10 \mu \mathrm{L}$ reaction mixtures with $2.5 \times$ HRM master mix (BioFire Defense, Salt Lake City, UT. USA) were PCR amplified for 55 cycles: $95{ }^{\circ} \mathrm{C}$ for $30 \mathrm{~s}, 66{ }^{\circ} \mathrm{C}$ for $30 \mathrm{~s}$, and $74{ }^{\circ} \mathrm{C}$ for $30 \mathrm{~s}$, followed by a pre-melt step of 95 and $28{ }^{\circ} \mathrm{C}$ for $30 \mathrm{~s}$ each. Products were melted from 45 to $90{ }^{\circ} \mathrm{C}$ on a BioFire Defense LightScanner-384 or -32 and analysed using the manufacturer software. Two plasmid controls containing the wild-type and mutant alleles were included as standards for every HRM run. Molecular barcoding was performed as described $[24,25]$ to identify monogenomic samples with unique parasite genotypes.

\section{Whole genome sequences}

This publication uses sequencing data generated by the Pf3k project [26]. The variant call files generated from this project were used to identify SNPs in each of the samples used in this study.

\section{Pfkelch13 PCR sequencing strategy}

The propeller domain of pfkelch13 was PCR amplified using Phusion HF DNA Polymerase kit and primers $3 F$ and $1 R^{\prime}$ in all 68 culture adapted parasites. DNA from KH001_024 was also amplified with primers 4F and $3 R^{\prime}$. An aliquot of PCR product was resolved by gel electrophoresis to check for specificity and yield and the remaining product was purified using DNA Clean \& Concentrator, ZymoResearch and sequenced using the same primers used to amplify the product by Genewiz. The full ORF of pfkelch13 was PCR amplified using primers $1 \mathrm{~F}$ and $1 \mathrm{R}$ from $3 \mathrm{D} 7$ and individual culture-adapted parasites. The resulting PCR product of $\sim 2.2 \mathrm{~kb}$ was purified by gel extraction (QIAquick Gel Extraction Kit, Qiagen) and sequenced at Genewiz using primers $1 \mathrm{~F}, 2 \mathrm{~F}, 3 \mathrm{~F}, 1 \mathrm{R}, 2 \mathrm{R}$ and $3 \mathrm{R}$. Primer sequences are as follows: 1F: 5'-ATGGAAGGAGAAAAAGTAA AAACAAAAGCAAATAG-3'; 2F: 5'-GGTAGGTGATTT AAGAATTACATTTATTAATTGGT-3'; 3F: $5^{\prime}$-CATTCC CATTAGTATTTTGTATAGGTG-3'; 4F: 5'-GTAGAGG TGGCACCTTTGAATACCCCTAGATCATC-3' 1R: 5' $^{\prime}$ T TATATAT T TGCTAT TAAAACGGAGTGACCAA ATCTG-3'; 1R': 5'-TTA TAT ATT TGC TAT TAA AAC GGA GTG-3'; 2R: 5'-AGCCTTATAATCATAGTTATTA CCACCAAAAACG-3'; 3R: 5'-TGTTGGTATTCATAAT

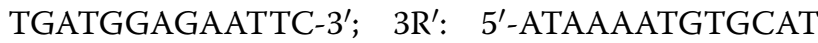
GAAAATAAATATTAAAG- ${ }^{\prime}$.

\section{In vitro 72-h drug susceptibility by SYBR green staining}

Drug susceptibility was measured using the SYBR Green I method as previously described [27]. Briefly, tightly synchronized $0-6 \mathrm{~h}$ rings were grown for $72 \mathrm{~h}$ in the presence of different concentrations of drugs in 384-well plates at $1 \%$ haematocrit and $1 \%$ starting parasitaemia; and, growth at $72 \mathrm{~h}$ was measured by SYBR Green staining of parasite DNA. Except for PPQ and KH001_053 where a 24-point dilution series was used, a 12-point dilution series of each drug was carried out in triplicate and repeated with at least three biological replicates. DMSO stocks of drugs were dispensed by a HP D300 Digital Dispenser (Hewlett Packard Palo Alto, CA, USA) except for the CQ and PPQ stocks that were prepared in water and dispensed with a Velocity 11 Robot (Bravo). Relative fluorescence units (RFU) was measured at an excitation of $494 \mathrm{~nm}$ and emission of $530 \mathrm{~nm}$ on a SpectraMax M5 (Molecular Devices Sunnyvale, CA, USA) and analysed using GraphPad Prism version 5 (GraphPad Software La Jolla, CA. USA). $\mathrm{EC}_{50}$ values were determined with the curve-fitting algorithm log(inhibitor) vs response-variable slope, except for PPQ and KH001_053. Due to the bimodal dose response of KH001_053 to PPQ, curve fitting didn't give an accurate $\mathrm{EC}_{50}$ value. The reported PPQ $\mathrm{EC}_{50} \mathrm{~s}$ for KH001_053 are estimates using biphasic curve 
fitting. Spearman correlation analysis was performed to assess the relationship between the anti-malarial $\mathrm{EC}_{50}$ values and in vivo clearance half-life, ring survival assay value or $p f m d r 1$ copy number. $\mathrm{p}$ values $<0.05$ were considered significant.

\section{Copy number variation assays}

To determine copy numbers for pfmdr1, plasmepsin II and the $63 \mathrm{~kb}$ amplicon genes (PF3D7_0520100, PF3D7_0520500, PF3D7_0520600, PF3D7_0520900 and PF3D7_0521000), real time quantitative PCR was performed on genomic DNA (extracted with QIAmp Blood Mini Kit, Qiagen) as previously described [28] with the following modifications: Amplification reactions were done in MicroAmp 384-well plates in $10 \mu \mathrm{L}$ SYBR Green master mix (Applied Biosystems), $150 \mathrm{nM}$ of each forward and reverse primer and $0.4 \mathrm{ng}$ template. Forty cycles were performed in the Applied Biosystems $\mathrm{ViiA}^{\mathrm{TM}}$ 7 Real-time PCR system (Life Technologies). Forward and reverse primers used were as previously described to amplify the following loci: pfmdr1 (PF3D7_0523000) [29], the $63 \mathrm{~kb}$ region on chromosome 5 (PF3D7_0520100, PF3D7_0520500, PF3D7_0520600, PF3D7_0520900 and PF3D7_0521000 [20]) and plasmepsin II (PF3D7_140800) [22]. For the endogenous controls, $\beta$-tubulin forward and reverse primers [28] were used for pfmdr1, PF3D7_0520100 and PF3D7_0520900 while pfldh forward and reverse primers [30] were used for PF3D7_0520500, PF3D7_0520600 and PF3D7_0521000. Target primers used were validated to have the same PCR efficiencies as their endogenous control primers; and, average copy number values were calculated for each gene using data from three independent experiments.

\section{Sequencing the pfort locus}

The entire pfcrt locus was sequenced as previously described [20] with some modifications. Briefly, total RNA was extracted using RNeasy kit (Qiagen) and used to generate cDNA using Superscript III (Invitrogen). The resulting cDNA was then used as template for PCR amplification of $p f c r t$ [20], followed by Sanger sequencing (GENEWIZ) [20]. Sequence data analysis was performed using MacVector.

\section{Ring survival assay $\left(\mathrm{RSA}_{\mathbf{0}-3 \mathrm{~h}}\right)$}

The $\mathrm{RSA}_{0-3 \mathrm{~h}}$ was performed as described previously [6]. Essentially, parasites were sorbitol synchronized twice at 40-h intervals, synchronous 40-44 h segmented schizonts were incubated for $15 \mathrm{~min}$ at $37{ }^{\circ} \mathrm{C}$ in serum-free media supplemented with heparin to disrupt agglutinated erythrocytes and late stages were purified with 35/65\% discontinuous Percoll gradient. The segmented schizonts were washed and cultured with fresh RBCs for $3 \mathrm{~h}$, after which late stages were removed by sorbitol treatment. Cultures with $0-3 \mathrm{~h}$ rings were adjusted to $2 \%$ haematocrit and $1 \%$ parasitaemia and seeded into a 24-well plate with $1 \mathrm{ml}$ complete media per well. To these wells, either DHA at $700 \mathrm{nM}$ or $0.1 \%$ DMSO were added immediately and incubated for $6 \mathrm{~h}$ at $37^{\circ} \mathrm{C}$, washed and incubated in drug free media. At $72 \mathrm{~h}$ from seeding, thin blood smears were made from control and treated wells and survival rates were measured microscopically by counting the proportion of next generation viable rings with normal morphology. Survival rates were expressed as ratios of viable parasitaemias in DHA-exposed and DMSO-treated controls. Parasites were counted from $10,000 \mathrm{RBC}$, and two separate individuals served as independent slide readers.

\section{Results}

Characteristics of original and culture-adapted parasite isolates

A total of 157 cryopreserved TRAC study samples collected in 2011 from Pursat or Pailin, Cambodia where ART resistance is observed were obtained. These isolates were selected before the discovery of the pfkelch13 marker and were designed to comprise western Cambodian samples, with two-thirds of the samples coming from the upper segment of the clearance half-life distribution and one-third being isolates with the shortest clearance times. 68 parasites were culture-adapted for further investigation, without knowledge of their in vivo clearance data. A summary of the overall population and the sub-set selected for culture-adaptation and further characterization is provided in Additional files 2 and 3. Collectively, $63 \%$ of both the original and the adapted parasites were from Pursat and the remaining 37\% were from Pailin (Additional file 3). Similarly, 67\% of both parasite sets (original and adapted) had a delayed clearance of $\geq 5 \mathrm{~h}$ and $33 \%$ had a clearance half-life of $<5 \mathrm{~h}$; for one isolate there were insufficient data to determine an in vivo half-life. All culture-adapted samples were confirmed to harbour monogenomic infections by molecular barcode analysis [25], only those parasites with unique barcode signatures [25] were initially pursued to maximize genetic diversity and exclude highly similar parasites among the analysed isolates.

\section{Pfkelch 13 propeller mutations are associated with parasite clearance half-life $\geq 5 \mathrm{~h}$}

Next, parasites were genotyped for the pfkelch13 locus previously associated with delayed parasite clearance (defined as $>5 \mathrm{~h}$ ) and increased ring-stage survival (defined as $\mathrm{RSA}_{0-3 \mathrm{~h}} \geq 1 \%$ ) [7]. An HRM genotyping assay $[24,25]$ for the common non-synonymous mutation conferring the C580Y change in PfKelch13 was developed 
and validated, among the 157 original samples with an unambiguous genotyping call, $71 \%(108 / 152)$ had the C580Y mutation (Additional file 2). These data, confirmed a significant positive association between the C580Y non-synonymous mutation and in vivo parasite clearance half-life values for these parasites [26]. This association was more pronounced among Pursat parasites (unpaired Student's t test with Welch's correction, $\mathrm{p}<0.0001)$ as compared to parasites from Pailin $(\mathrm{p}=0.0322$, Additional file 4$)$ for this sample set.

A combination of available whole genome sequence (WGS) data from the original samples [26, 31, 32], and pfkelch13-specific PCR-based sequencing identified both pfkelch13 mutations other than C580Y in this population and confirmed persistence of all pfkelch13 mutations in the culture adapted parasites further analysed. Overall there was good concordance between the molecular barcode derived from filter paper and cultured parasite samples, and between the pfkelch13 mutations identified in WGS or PCR re-sequencing data. Six different mutations were present in the pfkelch13 propeller domain: $\mathrm{Y} 493 \mathrm{H}$, R539T, I543T, C580Y, D584V, and H719N, and two pfkelch13 mutations outside the propeller region: $\mathrm{H} 136 \mathrm{~N}$, E270K. The two parasites with the H136N change also contained C580Y, but E270K was seen by itself, in a single isolate (Additional file 2). All propeller domain mutations were positively associated with in vivo clearance values (Fig. 1a), including parasites with the double mutation H136N/C580Y genotype (Additional file 2). Additionally, two asparagine (Asn) insertions at codon 142 were identified in all isolates tested except those with the $\mathrm{Y} 493 \mathrm{H}$ change and one parasite with the D584V genotype (Additional file 2). The location of this Asn insertion was distinct from that previously noted among Asian parasites [33]. No association between this Asn insertion and either parasite clearance half-life or $\mathrm{RSA}_{0-3 \mathrm{~h}}$ phenotype was detected.

The sub-set of 68 culture-adapted parasites harbored all mutation types observed in the 157 initial isolates except $\mathrm{H} 136 \mathrm{~N}$, and each pfkelch13 mutation detected by WGS was confirmed by PCR re-sequencing (Additional files 2,3). Overall, the sub-set of culture-adapted parasites showed similar distribution of pfkelch 13 genotypes detected among the original 157 isolates chosen for this study. The majority of parasites in this sub-set $(79 \%$ or 54/68) carried non-synonymous mutations in pfkelch13, with the most common change resulting in C580Y (53\% or $36 / 68$, Table 1 ). Among these culture-adapted parasites from Pailin there was a higher frequency of pfkelch13 mutations than parasites from Pursat (88 and $74 \%$, respectively), with the $\mathrm{C} 580 \mathrm{Y}$ mutation being the most prevalent (77 and 60\%, respectively). Both
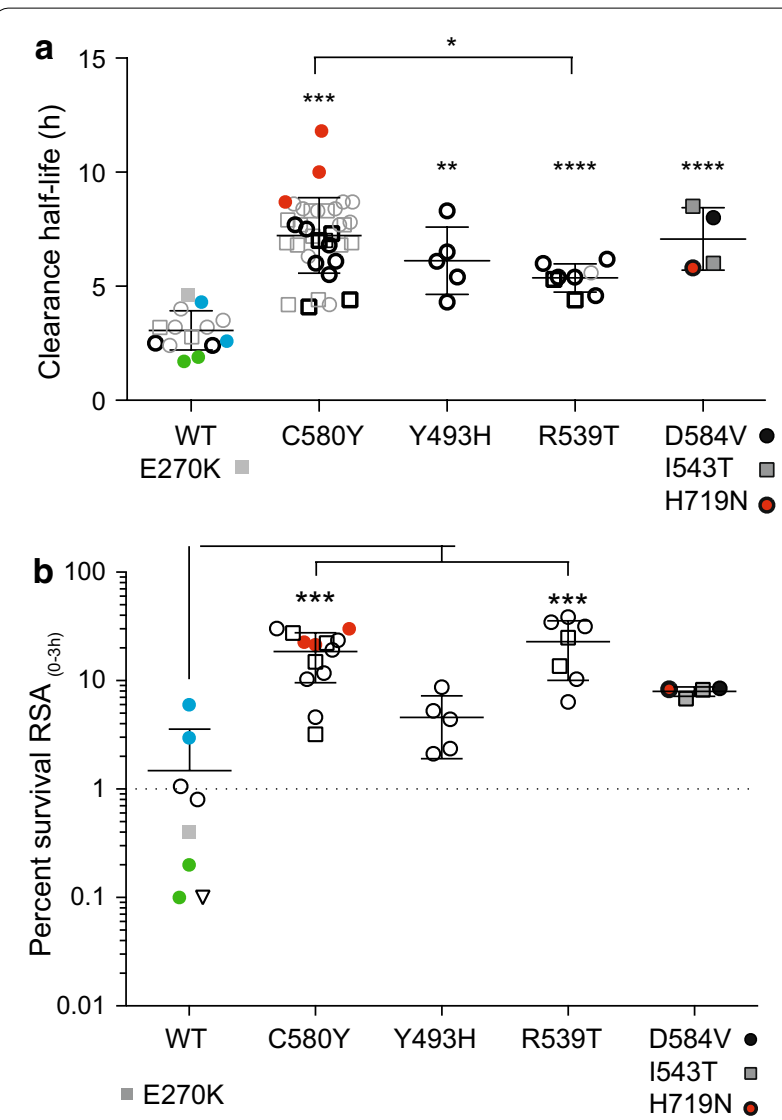

Fig. 1 Positive association between pfkelch13 propeller mutations and in vivo clearance half-life as well as in vitro $\mathrm{RSA}_{0-3 \mathrm{~h}}$. Parasites from Pursat (round symbo/s) or Pailin (square symbols) were classified according to their pfkelch13 alleles. a Comparison of clearance half-live values (hours, h) from culture-adapted parasites harbouring different pfkelch 13 propeller alleles, and combining alleles represented by less than five samples. Parasites outlined in bold were further tested for $\mathrm{RSA}_{0-3 \mathrm{~h}}$ phenotypes in $\mathbf{b}$. A one-way ANOVA test, with a Tukey's post-test, was performed between wild-type (WT) and each allele category. Significance values are indicated by asterisks: ${ }^{*}(p<0.05) ;{ }^{* *}(p<0.01) ;{ }^{* *}(p<0.001) ;$ and ${ }^{* * *}(p<0.0001)$. b In vitro $\mathrm{RSA}_{0-3 \mathrm{~h}}$ testing. Percent survival is displayed on the $y$-axis and pfkelch 13 allele represented on the $x$-axis. All parasites that harbour a pfkelch 13 propeller mutation exhibit an $\mathrm{RSA}_{0-3 \mathrm{~h}}$ value of $>1 \%$ (dotted line). Data were analysed by Kruskal-Wallis test followed by Dunn's multi-comparison test. p values: ${ }^{* * *}<0.001$. An additional subset represented by coloured symbols, also underwent conventional in vitro drug testing and assessment of pfmdr1 copy number variation. These were chosen as representatives of parasites that were ART sensitive (green) or, ART resistant (red) as assessed by both $\mathrm{RSA}_{0-3 h}$ phenotype and pfkelch13. Two 'discordant' samples (resistant according to RSA but wild-type at pfkelch13) are indicated in blue

geographic locations had their own private mutations not found in the other location (Additional file 2). All pfkelch13 propeller mutations displayed positive associations with in vivo clearance phenotypes (Fig. 1a). 
Table 1 Summary of PfKelch13 mutations

\begin{tabular}{lccccccccccc}
\hline Culture adapted parasites & Total & WT & Y493H & R539T & I543T & C580Y & D584V & H719N & H136N/C580Y & E270K & Asn Insert \\
\hline Pailin & 25 & 3 & 0 & 2 & 2 & 17 & 0 & 0 & 0 \\
Pursat & 43 & 11 & 5 & 6 & 0 & 19 & 1 & 1 & 0 \\
Total & 68 & 14 & 5 & 8 & 2 & 36 & 1 & 1 & 0 \\
\hline
\end{tabular}

Pfkelch13 propeller mutations are positively associated with increased ring-stage survival by in vitro $\mathrm{RSA}_{\mathbf{0 - 3 h}}$

There was a positive association between increased $\mathrm{RSA}_{0-3 \mathrm{~h}}$ survival phenotypes and pfkelch13 mutations among 36 culture adapted parasites (Fig. 1b) and parasites with the D584V change previously associated with increased in vivo parasite clearance time [5] demonstrated an increased ring-stage survival by $\mathrm{RSA}_{0-3 \mathrm{~h}}$.

In vitro $\mathrm{RSA}_{0-3 \mathrm{~h}}$ phenotypes were evaluated as a measure of ART resistance for 36 adapted parasites exhibiting a range of in vivo clearance phenotypes and harbouring a variety of pfkelch13 mutations. Consistent with previous reports [6], the distribution of $\mathrm{RSA}_{0-3 \mathrm{~h}}$ phenotypes spanned two $\log _{10}$ ranges for all parasites analysed. Overall, there was a significant difference between wild-type $\left(\mathrm{RSA}_{0-3 \mathrm{~h}}=0.1-6.0 \%, \mathrm{n}=7\right)$ parasites and those with pfkelch13 mutations $\left(\mathrm{RSA}_{0-3 \mathrm{~h}}=2.1-38.6 \%, \mathrm{n}=29\right)$ in terms of $\mathrm{RSA}_{0-3 \mathrm{~h}}$ parasite survival rate (Student's $\mathrm{t}$ test $\mathrm{p}=0.0018)$. $\mathrm{RSA}_{0-3 \mathrm{~h}}$ survival rates also differed between parasites with individual pfkelch13 mutations; whereby parasites with either a C580Y or R539T mutation exhibited the highest survival rates (Fig. 1b). One isolate with a PfKelch13 E270K mutation outside the propeller domain exhibited a clearance of $>4 \mathrm{~h}$, yet had an $\mathrm{RSA}_{0-3 \mathrm{~h}}$ value of $0.4 \%$. Among the parasites with $\mathrm{RSA}_{0-3 \mathrm{~h}}$ values $>1 \%, 19 \%$ $(7 / 36)$ had in vivo clearance values between 4 and $5 \mathrm{~h}$, which suggests that for this specific population a clearance value of $4 \mathrm{~h}$ or greater is more consistent with ART resistance based upon an $\mathrm{RSA}_{0-3 \mathrm{~h}}$ phenotype (Additional file 5).

All pfkelch13 propeller polymorphisms found among these parasites were previously identified [7, 33]. However, a novel positive association was demonstrated between the $\mathrm{D} 584 \mathrm{~V}$ mutation and in vitro ring survival values ( $8.0 \mathrm{~h}$ clearance and $8.5 \%$ RSA survival for $\mathrm{D} 584 \mathrm{~V})$, not previously tested for in vitro ART resistance (Fig. 1b). These data confirm that all pfkelch13 propeller mutations found in these TRAC isolates are associated with an $\mathrm{RSA}_{0-3 \mathrm{~h}}$ phenotype $\geq 1 \%$, thus considered in vitro ART resistant.

Identification of discordant parasites that exhibit increased ring-stage survival but lack Pfkelch 13 mutations

ART resistance in vitro has been suggested to correlate with an $\mathrm{RSA}_{0-3 \mathrm{~h}}$ survival value of $\geq 1 \%$ [6], and all isolates in this study with a mutation in pfkelch13 had an $\mathrm{RSA}_{0-3 \mathrm{~h}} \geq 1 \%$. All wild-type pfkelch13 parasites had in vivo clearance half-lives below $5 \mathrm{~h}$ (Additional file 2); however, there was a range of in vitro $\mathrm{RSA}_{0-3 \mathrm{~h}}$ survival phenotypes from 0.1 to $6 \%$, including four isolates with $\mathrm{RSA}_{0-3 \mathrm{~h}}$ of approximately $\geq 1 \%(0.8,1.1,3$, and $6 \%)$ (Fig. 1b). Parasites with an $\mathrm{RSA}_{0-3 \mathrm{~h}}$ of $\geq 0.8 \%$ that lack pfkelch13 mutations are considered discordant. These data suggest changes outside pfkelch13 could also confer an increased ring-stage survival phenotype indicative of ART resistance in this parasite population.

Using WGS data previously generated [26] for 33 of the $\mathrm{RSA}_{0-3 \mathrm{~h}}$ phenotyped parasites, the question was explored whether mutations outside pfkelch13 were evident in the four parasites with $\mathrm{RSA}_{0-3 \mathrm{~h}} \geq 0.8 \%$ values that lack pfkelch13 ORF mutations. This small data set was underpowered to identify novel mutations using a genome-wide strategy, but a candidate gene approach was undertaken to ask whether any known drug resistant loci or other previously identified secondary mutations could potentially explain the high $\mathrm{RSA}_{0-3 \mathrm{~h}}$ survival values (Additional file 6). Variant positions were identified across 23 genes previously associated with ART resistance among the set of $33 \mathrm{RSA}_{0-3 \mathrm{~h}}$ phenotyped parasites $[7,15,26]$, which included both sensitive and resistant parasites. Examination of both sensitive and resistant parasites would allow detection of mutations that may account for the differences in $\mathrm{RSA}_{0-3 \mathrm{~h}}$ survival values and identification of mutations that confer high $\mathrm{RSA}_{0-3 \mathrm{~h}}$ survival values independent of pfkelch13.

This analysis identified 37 SNPs (30 non-synonymous, four synonymous, two intronic) spread across 19 unique genes (Additional file 7). Eleven of these positions were invariant among these four isolates that lack pfkelch13 ORF mutations but exhibited an $\mathrm{RSA}_{0-3 \mathrm{~h}}$ survival value $\geq 0.8 \%$. These 37 SNP positions are found in genes known to be associated with drug resistance including dihydrofolate reductase (dhfr, PF3D7_0417200) [34] and dihydropteroate synthase (dhps, PF3D7_0810800) [35]. Another locus, phosphatidylinositol-4-phosphate 5-kinase (PIP5K, PF3D7_0110600) [36], is involved in cellular signaling pathways, including synthesis of PIP2, a substrate for PI3K to produce PIP3, which activates the AKT family of serine/threonine kinases, a pathway implicated in an ART resistance mechanism [15]. The 
putative NLI Interacting factor-like phosphatase (NIF4, PF3D7_1012700) [36-39] is a protein phosphatase 2C protein. Several members of the Plasmodium phosphatome contain kelch domains and have been shown to be key regulators of parasite development and differentiation [37, 40], but NIF4 lacks an intact $\operatorname{DxDx}(\mathrm{T} / \mathrm{V})$ motif, and thus may be catalytically inactive [39]. Pfmdr2 (PF3D7_1447900) [41, 42] is implicated as a secondary locus associated with delayed parasite clearance [26]. No other changes associated with ART resistance reported in the literature $[43,44]$ were detected among these parasites (Additional file 6).

\section{Detection of $P P Q$ resistance phenotype}

To further evaluate the relationship between $\mathrm{RSA}_{0-3 \mathrm{~h}}$ phenotype and pfkelch13 mutations, a sub-set of eight isolates with a range of ring-stage survival phenotypes was selected. This sub-set included the two most sensitive (in green) and the two most resistant (discordant, in blue) parasites without pfkelch13 mutations, as well as four resistant parasites that contain pfkelch13 mutations (in red) (Fig. 1b). First standard in vitro drug tests [27] were used to evaluate anti-malarial drug responses among these parasites (Fig. 2). $\mathrm{EC}_{50}$ values for ART and its derivatives did not consistently correlate with either the in vivo clearance or in vitro $\mathrm{RSA}_{0-3 \mathrm{~h}}$ phenotypes, as previously reported [6, 7] (Additional file 8; Fig. 2). The parasite clearance half-life and $\mathrm{RSA}_{0-3 \mathrm{~h}}$ survival phenotypes did not consistently associate with responses to other anti-malarial compounds tested (chloroquine $(\mathrm{CQ})$, MFQ, lumefantrine (LUM), atovaquone (ATV), quinine (QN) and PPQ, Additional file 8). A significant positive correlation was observed between $\mathrm{EC}_{50}$ values for ART $(\mathrm{r}=0.881, \mathrm{p}=0.0072)$, artesunate $(\mathrm{AS}, \mathrm{r}=0.719$, $\mathrm{p}=0.0368)$, MFQ $(\mathrm{r}=0.7619, \mathrm{p}=0.0368)$ and LUM $(\mathrm{r}=0.857, \mathrm{p}=0.0107)$ and increased $p f m d r 1$ copy number (Spearman correlation, Additional file 8 and Fig. 2), consistent with previous reports [30, 45, 46].

Interestingly, one isolate, KH001_053, with a slow clearance time $(8.7 \mathrm{~h})$ and high ring survival $(30 \%)$
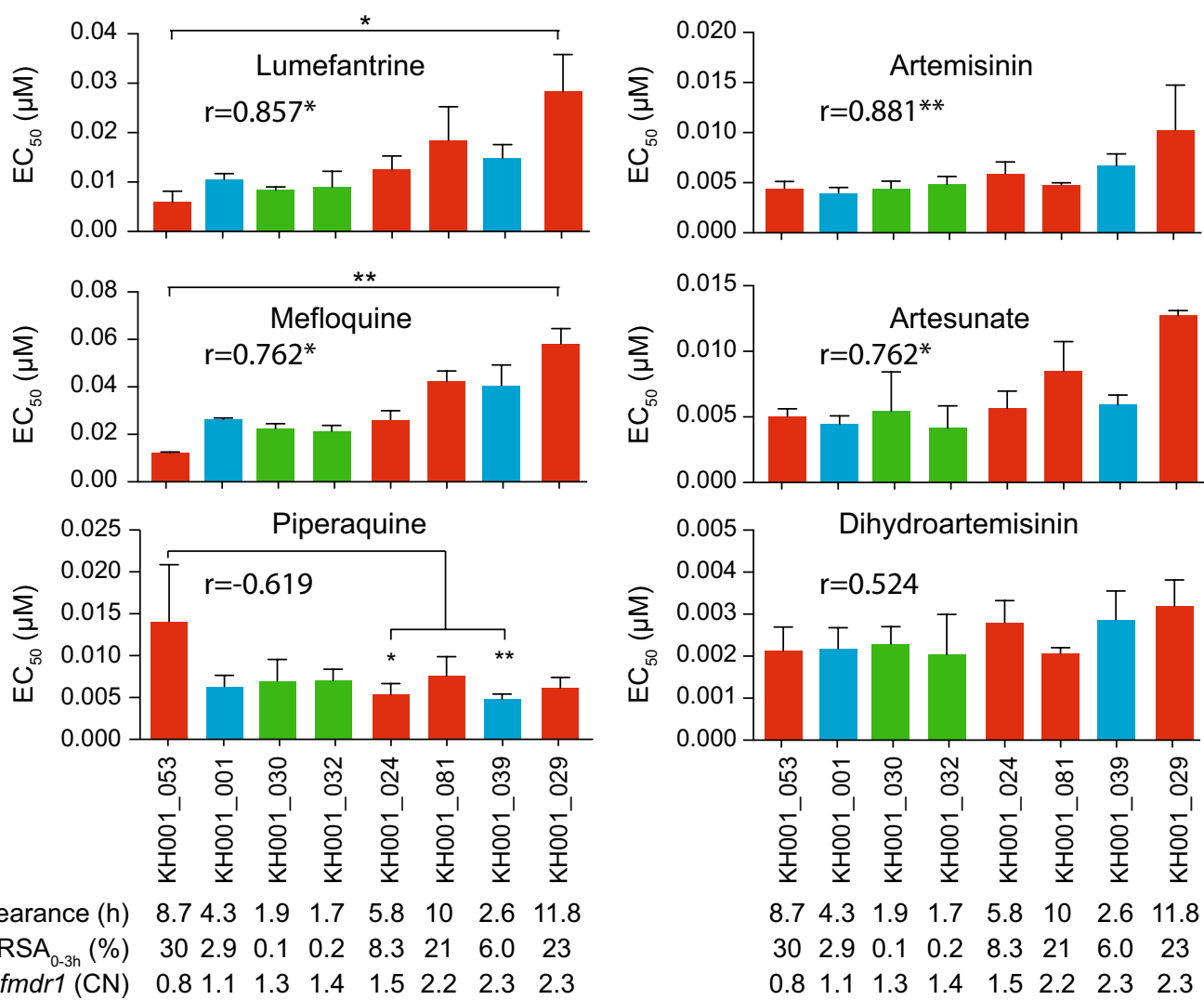

Fig. 2 Pfmdr 1 copy numbers are positively associated with MEF, LUM, ART and ARS. Drug sensitivity assays were set up with 0-6 h old ring stage parasites and \% growth was measured $72 \mathrm{~h}$ later by SYBR Green. Shown are the average $\mathrm{EC}_{50}$ values from at least three biological replicates for two ART sensitive (green), two discordant (blue) and four resistant (red) isolates. Their respective clearance time, RSA ${ }_{0-3 h}$ percent survival and their pfmdr 1 copy number are indicated at the bottom. The Spearman correlation coefficient for pfmdr 1 copy number and each drug is shown in the graph. A one-way ANOVA test, with a Tukey's post-test was performed between all isolates for each drug. Significance values are indicated by asterisks, as follows: ${ }^{*}(p<0.05){ }^{* *}(p<0.01)$ 

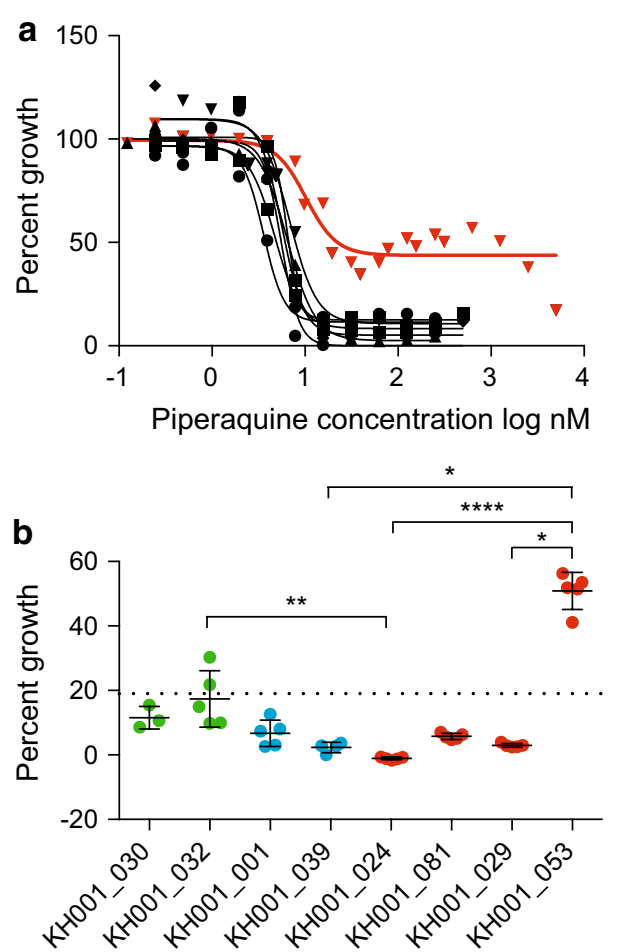

Fig. 3 KH001_053 exhibits resistance to piperaquine (PPQ). a EC 50 growth curves for eight parasite isolates in different PPQ concentrations. Percent growth is displayed on the $y$-axis and the PPQ concentration $(\mathrm{nM})$ is represented in a log scale on the $\mathrm{x}$-axis, with KH001_053 indicated by red triangles. b Relative growth is shown (percent growth) for TRAC isolates exposed to $125 \mathrm{nM} P \mathrm{PQ}$ for $72 \mathrm{~h}$ starting with $0-6 \mathrm{~h}$ old rings, with individual parasite lines represented on the $x$-axis. Colours correspond to parasites with $\mathrm{RSA}_{0-3 \mathrm{~h}} \leq 0.2 \%$ that lack pfkelch 13 mutations (sensitive, green); $\mathrm{RSA}_{0-3 \mathrm{~h}}>3 \%$ that lack pfkelch 13 mutations (discordant, blue); and, $\mathrm{RSA}_{0-3 \mathrm{~h}}>1 \%$ that harbour with pfkelch 13 mutations (resistant, red). Data were analysed by Kruskal-Wallis test followed by Dunn's multicomparison test. p values: ${ }^{*}<0.1,{ }^{* *}<0.01,{ }^{* * * *}<0.0001$

exhibited a bimodal response to PPQ that indicated parasite survival at high PPQ concentrations (Fig. 3a). Compared to other isolates, KH001_053 also demonstrated a high survival phenotype (50-70\%) when grown at high concentrations of PPQ (125 and $250 \mathrm{nM}$ ) for $72 \mathrm{~h}$ [47] (Fig. 3b). This PPQ-resistant parasite also demonstrated hyper-susceptibility to MFQ and LUM (Fig. 2), explicable at least in part by a single copy of the $p f m d r 1$ gene (Additional file 8).

KH001_053 was then tested for evidence of known molecular markers associated with PPQ resistance [20], including C101F mutation in pfcrt, an amplification of $63 \mathrm{~kb}$ region on chromosome 5, and amplification of plasmepsin II [21, 22]. All eight isolates were evaluated for SNPs in the pfcrt locus by PCR amplification and sequencing of cDNA across pfcrt. Eight non-synonymous mutations (M74I, N75E, K76T, A220S, Q271E, N326S, I356T, R371I) were found that were fixed in these eight isolates. No evidence of the previously reported C101F haplotype in the KH001_053 parasite was detected [20]. Since PPQ resistance has been associated with an increased copy number of a $63 \mathrm{~kb}$ amplicon on chromosome 5 (including PF3D7_0520100, PF3D7_0520500, PF3D7_0520600, PF3D7_0520900 and PF3D7_0521000, $825600-888300 \mathrm{bp}$ ) and a decreased number of $p f m d r 1$ in vitro $[20,30]$ as well as an increased copy number of plasmepsin II (PF3D7_140800) locus in vivo [21, 22], copy number assays were performed on those loci. In concordance with to a previous study [30] no increased copy number on the $63 \mathrm{~kb}$ amplicon region on chromosome 5 was detected. However, KH001_053 did harbour two copies of plasmepsin II consistent with previous reports where the presence of an increased plasmepsin II and III copy number was predictive of recrudescence in DHA/PPQ-treated patients [21, 22]. These findings strengthen the hypothesis that increased plasmepsin II copy number may play a role in PPQ resistance.

\section{Discussion}

Within a set of 68 culture-adapted Cambodian parasites, some parasites exhibited an increased $\mathrm{RSA}_{0-3 \mathrm{~h}}$ phenotype that lack pfkelch13 mutations, a parasite harbouring the PfKelch13 D584V change exhibited increased $\mathrm{RSA}_{0-3 \mathrm{~h}}$ survival, and some parasites showed evidence for PPQ resistance. To evaluate a set of 157 Cambodian P. falciparum isolates from the TRAC study, with known clearance half-life data, a genotyping assay for the common C580Y change associated with ART resistance was developed and confirmed a clear association between delayed parasite clearance half-life and the presence of the C580Y allele. Sequence or genotype data from 146 of these parasites demonstrated a positive association between the clearance phenotype and pfkelch13 propeller mutations. 68 of these parasites were culture-adapted and 36 were tested for their in vitro $\mathrm{RSA}_{0-3 \mathrm{~h}}$ phenotype. Increased parasite clearance half-life was associated with $\mathrm{RSA}_{0-3 \mathrm{~h}} \geq 1 \%$ values, with a 4 -h or greater clearance phenotype being more consistent with ART resistance based upon $\mathrm{RSA}_{0-3 \mathrm{~h}}$ for this population. A novel positive association was demonstrated between $\mathrm{D} 584 \mathrm{~V}$ and in vitro ring-stage parasite survival under DHA among these parasites.

Despite this general correspondence between in vivo clearance and in vitro $\mathrm{RSA}_{0-3 \mathrm{~h}}$ phenotypes and Pfkelch13 propeller mutations, there were discordant parasites that exhibited a resistant $\mathrm{RSA}_{0-3 \mathrm{~h}}$ phenotype $\left(\mathrm{RSA}_{0-3 \mathrm{~h}} \geq 0.8 \%\right)$ yet harboured no mutations in the entire pfkelch13 
ORF, consistent with other studies that found similarly discordant parasites by $\mathrm{RSA}_{0-3 \mathrm{~h}}$ [48], or by clearance half-life [26]. A parasite (see Additional file 5) with a PfKelch13 E270K outside the propeller domain exhibited a clearance value of $>4 \mathrm{~h}$, yet had an $\mathrm{RSA}_{0-3 \mathrm{~h}}$ value of $0.4 \%$. These differences may be due to differential host factors including variances in drug pharmacokinetics, pharmacodynamics or host immune status. More isolates with an E270K mutation need to be studied to confirm the role of a pfkelch13 mutation outside the propeller domain in ART resistance and reduce the probability of an artifact related to microscopy. Taken together, these results suggest that ART resistance in natural parasite populations could be mediated by changes outside of the pkelch13 propeller domain. Parasites with these phenotypes around the defined cut off values may be important for identification and study of loci other than pfkelch 13 that contribute to ART resistance. Inspection of candidate loci, including traditional drug resistance mutations, recently identified secondary mutations, or loci related to pathways implicated in ART resistance, identified a few specific changes that may account for these discordant parasites, but lacked the statistical power to determine whether any of these contributed to ART resistance. However, identification of potential mutations in the PIP5K and $p f m d r 2$ loci is consistent with other reports. Nevertheless, the identification of parasites that lack pfkelch13 mutations yet harbour an increased $\mathrm{RSA}_{0-3 \mathrm{~h}}$ survival phenotype, suggest loci other than pfkelch13 may modulate ART resistance in these parasites. Alternative strategies, such as the use of independent chemogenomic strategies or genetic crosses in vitro [49], might be useful to identify loci involved in conferring the observed increased ring-stage survival phenotype in the absence of pfkelch13 mutations.

No relationship was found between $\mathrm{EC}_{50}$ values for ART or its derivatives with either clearance or ring-stage survival phenotypes, but a previously noted correspondence was confirmed between $p f m d r 1$ copy number and $\mathrm{EC}_{50}$ values of ART and AS, as well as with MFQ and LUM responses, among eight parasites evaluated for drug responses (Fig. 2). Drug testing identified a PPQresistant isolate among this population, consistent with reports in the literature of increased PPQ resistance in this region $[18,19]$. The PPQ-resistant parasite had a single copy of the $p f m d r 1$ gene $[20,30]$ and amplification of the plasmepsin II locus previously noted [21, 22]. The exact nature of this resistance, the role of plasmepsin II and the explanation for the apparent bimodal response to PPQ remains unknown and under investigation, but identification of culture-adapted parasites affords additional testing of both the phenotype and genotype (Additional file 1).

It has been suggested $[18,19]$ that PPQ resistance arose in the context of or as a consequence of ART resistance. Understanding the nature of this resistance and the underlying mechanism will be critical for reducing or restricting emergence of PPQ resistance in Southeast Asia where reports indicate rapid emergence over the past several years [18]. Furthermore, since DHA-PPQ is being utilized in mass drug administration campaigns designed to facilitate elimination of malaria in specific settings, use of molecular markers of resistance and understanding the relationship between ART and PPQ resistance will be important. Thus, identification of PPQ resistance with MFQ and LUM hyper-susceptibility in an ART resistant parasite could be an important clue to understanding mechanisms of drug response, and testing the implications of triple therapy, such as ART-PPQMFQ, being considered for use in this region [18].

\section{Conclusions}

In a large set of $P$. falciparum isolates from the TRAC study, the associations between C580Y and several other pfkelch 13 propeller mutations and parasite clearance half-life was investigated. In a subset of 68 cultureadapted parasites, $\mathrm{RSA}_{0-3 \mathrm{~h}}$ survival and conventional responses to multiple antimalarial drugs were measured. Several pfkelch13 mutations (including D584V) were associated with increased $\mathrm{RSA}_{0-3 \mathrm{~h}}$ survival, and discordant parasites with $\mathrm{RSA}_{0-3 \mathrm{~h}}$ survival $1 \%$ but without pfkelch13 ORF mutations were identified. These data suggest that mutations outside of Pfkelch13 may confer in vitro ART resistance in $P$. falciparum. It will therefore be important to continue phenotypic assessment of ART resistance, in addition to surveying for pfkelch13 propeller mutations. Detection of a PPQ-resistant parasite will enable further studies to investigate underlying mechanisms of PPQ resistance. This panel of culture-adapted parasites with known parasite clearance half-life, $\mathrm{RSA}_{0-3 \mathrm{~h}}$ survival, and pfkelch13 genotype will facilitate further investigation of ART resistance mechanisms, providing tools to identify potential PfKelch13-binding partners and other interacting molecules. 


\section{Additional files}

Additional file 1. Culture-adaptation of TRAC parasites.

Additional file 2. Summary of all genotypes available.

Additional file 3. Schematic shows distribution of parasites obtained from Pursat (blue star on map, $\mathrm{KH} 1$ parasites indicated in blue) and Pailin (red star, $\mathrm{KH} 4$ parasites indicated in red) from Cambodia (map modified from https://commons.wikimedia.org/wiki/ File:Cambodia_provinces_en.svg).

Additional file 4. Distribution of C580Y mutations in Cambodian isolates. Parasites from Pursat (round symbols) or Pailin (square symbols) were classified according to their Pfkelch13 alleles. Comparison of clearance half-life values (hours, h) from parasites harboring the wild-type (C) or mutant (Y) allele at amino acid position 580 in the Pfkelch13 locus, with cultureadapted parasites represented by black outlined symbols. C580 parasites harboring a mutation in the Pfkelch13 propeller domain other than C580Y are indicated by grey filled symbols. An unpaired t test was performed between the C580 and C580Y carrying parasites from Pursat $(p<0.0001)$ or Pailin ( $p<0.05)$; with significant differences $(p<0.0001$ overall) when both Pursat and Pailin were combined.

Additional file 5. Association between percent survival $\mathrm{RSA}_{0-3 \mathrm{~h}}$ and clearance time. Correlation between clearance time on the $\mathrm{x}$-axis and percent survival $\mathrm{RSA}_{0-3 \mathrm{~h}}$ on the $\mathrm{y}$-axis. Each dot represents an isolate. Samples represented by color were used for in vitro drug testing and for pfmdr 1 copy number variation determination — green for ART sensitive; blue for discordant; and red for ART resistant parasites. The grey dot marks the isolate with an E270K mutation. The dotted lines represent the cut off used to discriminate between resistant and sensitive parasites ( $1 \%$ for $\mathrm{RSA}_{0-3 h^{\prime}} 4$ or $5 \%$ for clearance).

Additional file 6. All genes analysed for candidate gene approach. Additional file 7. All positions that are variant in $\mathrm{RSA}_{0-3 \mathrm{~h}}$ phenotyped isolates.

Additional file 8. Spearman correlation coefficients between antimalarial EC50 values, in vivo clearance half-life, ring survival assay ${ }_{0-3 h}$ or pfmdr 1 copy number.

\section{Abbreviations}

ACT: artemisinin combination therapy; ART: artemisinin; AS: artesunate; Asn: asparagine; ATV: atovaquone; CQ: chloroquine; DHA: dihydroartemisinin; HRM: high resolution melt; iRBC: infected red blood cell; LUM: lumefantrine; MFQ: mefloquine; NIF4: NLI interacting factor-like phosphatase; ORF: open reading frame; PCR: polymerase chain reaction; PPQ: piperaquine; QN: quinine; RBC: red blood cells; SNP: single nucleotide polymorphism; TRAC: Tracking Resistance to Artemisinin Collaboration; WGS: whole genome sequencing; $\mathrm{RSA}_{0-3 \mathrm{~h}}$ ring-stage survival assay; PIP5K: phosphatidylinositol-4-phosphate 5-kinase; CNV: copy number variation.

\section{Authors' contributions}

AM, SB, PM carried out $\mathrm{RSA}_{0-3 h^{\prime}}$ drug testing, PCR-based genotyping and copy number variation analysis and helped write the manuscript; WW carried out the genomic analysis with data provided by OM and helped write the manuscript; RD developed the C580Y HRM genotyping assay and carried out molecular barcoding of the samples; AD created primer sets and helped with $\mathrm{RSA}_{0-3 \mathrm{~h}}$ analysis of the samples; SS helped with genomic analysis and provided critical edits to the manuscript; CA, PL, MD, CW, EAA, AMD, NJW, and RF carried out field collection and provided in vivo sample information and samples. DFW provided critical review of the data and experimental guidance. SKV culture adapted the parasites, provided critical review of the data and experimental guidance, and wrote the manuscript. All authors provided critical review of the data and manuscript before publication. All authors read and approved the final manuscript.

\begin{abstract}
Author details
${ }^{1}$ Department of Immunology and Infectious Disease, Harvard T.H. Chan School of Public Health, 665 Huntington Avenue, I-704, Boston, MA 02115, USA. ${ }^{2}$ Infectious Disease Initiative, The Broad Institute of MIT and Harvard, Cambridge, MA, USA. ${ }^{3}$ Laboratory of Malaria and Vector Research, National Institute of Allergy and Infectious Diseases, National Institutes of Health, Rockville, MD, USA. ${ }^{4}$ Asia Regional Centre, Worldwide Antimalarial Resistance Network, Bangkok, Thailand. ${ }^{5}$ Nuffield Department of Medicine, Centre for Tropical Medicine and Global Health, Centre for Tropical Medicine and Global Health, University of Oxford, Oxford, UK. ${ }^{6}$ Mahidol-Oxford Tropical Medicine Research Unit, Mahidol University, Bangkok, Thailand. ${ }^{7}$ Centre for Genomics and Global Health, Wellcome Trust Centre for Human Genetics, University of Oxford, Oxford, UK. ${ }^{8}$ Wellcome Trust Sanger Institute, Hinxton, UK. ${ }^{9}$ Department of Intensive Care, Academic Medical Center, University of Amsterdam, Amsterdam, The Netherlands. ${ }^{10}$ School of Nursing and Health Sciences, Simmons College, Boston, MA, USA.
\end{abstract}

\section{Acknowledgements}

We would like to thank the patients who participated to the TRAC study and Dr. Kesinee Chotivanich and her team for initial cryopreservation of the isolates. We would like to thank Courtney Edison for help with tissue culture and RSA assays, and Elizabeth Hamilton for work developing the C580Y genotyping assay and for genotyping filter paper derived samples. We would also like to thank Katelyn Durfee for molecular barcode analysis of culture-adapted lines. We thank Paul Hinkson for his help with $\mathrm{EC}_{50}$ assays for anti-malarial drug testing of the culture-adapted parasites.

\section{Competing interests}

The authors declare that they have no competing interests.

\section{Availability of data and materials}

Datasets generated and/or analysed during the current study are available through the MalariaGEN Pf3K Project [26, 32].

\section{Ethics approval and consent to participate}

The scientific merit and use of human subjects for this study was approved by the institutional review board (IRB) of the Harvard T. H. Chan School of Public Health (22689-101). Written informed consent was obtained from all adult subjects and from the parent or legal guardians of minors.

\section{Funding}

We thank the generous funding that supported this work. The TRAC study was funded by the UK Department for International Development; support for culture-adaptation and analysis at the Harvard T. H. Chan School of Public Health was provided by the University of Oxford/Bill and Melinda Gates Foundation for "Identifying a Laboratory Marker of Artemisinin Resistance" (OPP104063, PI: Nicholas White); and from the Bill and Melinda Gates Foundation "Genomics-Based Diagnostics for Elimination and Eradication of Plasmodium" (OPP1053604, Pl: Dyann Wirth)

\section{Publisher's Note}

Springer Nature remains neutral with regard to jurisdictional claims in published maps and institutional affiliations.

Received: 26 December 2016 Accepted: 29 April 2017

Published online: 12 May 2017

\section{References}

1. Lubell Y, Dondorp A, Guerin PJ, Drake T, Meek S, Ashley E, et al. Artemisinin resistance-modelling the potential human and economic costs. Malar J. 2014;13:452.

2. White NJ. Artemisinin resistance - the clock is ticking. Lancet. 2010;376:2051-2.

3. Dondorp AM, Nosten F, Yi P, Das D, Phyo AP, Tarning J, et al. Artemisinin resistance in Plasmodium falciparum malaria. N Engl J Med. 2009;361:455-67. 
4. Noedl H, Socheat D, Satimai W. Artemisinin-resistant malaria in Asia. N Engl J Med. 2009;361:540-1.

5. Ashley EA, Dhorda M, Fairhurst RM, Amaratunga C, Lim P, Suon S, et al. Spread of artemisinin resistance in Plasmodium falciparum malaria. N Engl J Med. 2014:371:411-23.

6. Witkowski B, Amaratunga C, Khim N, Sreng S, Chim P, Kim S, et al. Novel phenotypic assays for the detection of artemisinin-resistant Plasmodium falciparum malaria in Cambodia: in vitro and ex vivo drug-response studies. Lancet Infect Dis. 2013;13:1043-9.

7. Ariey F, Witkowski B, Amaratunga C, Beghain J, Langlois AC, Khim N, et al. A molecular marker of artemisinin-resistant Plasmodium falciparum malaria. Nature. 2014;505:50-5.

8. Straimer J, Gnadig NF, Witkowski B, Amaratunga C, Duru V, Ramadani AP, et al. Drug resistance. K13-propeller mutations confer artemisinin resistance in Plasmodium falciparum clinical isolates. Science. 2015;347:428-31.

9. Ghorbal M, Gorman M, Macpherson CR, Martins RM, Scherf A, LopezRubio JJ. Genome editing in the human malaria parasite Plasmodium falciparum using the CRISPR-Cas9 system. Nat Biotechnol. 2014;32:819-21.

10. Hong $Y L$, Yang $Y Z$, Meshnick SR. The interaction of artemisinin with malarial hemozoin. Mol Biochem Parasitol. 1994;63:121-8.

11. Wang J, Zhang CJ, Chia WN, Loh CC, Li Z, Lee YM, et al. Haem-activated promiscuous targeting of artemisinin in Plasmodium falciparum. Nat Commun. 2015;6:10111.

12. Xie SC, Dogovski C, Hanssen E, Chiu F, Yang T, Crespo MP, et al. Haemoglobin degradation underpins the sensitivity of early ring stage Plasmodium falciparum to artemisinins. J Cell Sci. 2016;129:406-16.

13. Dogovski C, Xie SC, Burgio G, Bridgford J, Mok S, McCaw JM, et al. Targeting the cell stress response of Plasmodium falciparum to overcome artemisinin resistance. PLoS Biol. 2015;13:e1002132.

14. Mok S, Ashley EA, Ferreira PE, Zhu L, Lin Z, Yeo T, et al. Drug resistance. Population transcriptomics of human malaria parasites reveals the mechanism of artemisinin resistance. Science. 2015;347:431-5.

15. Mbengue A, Bhattacharjee S, Pandharkar T, Liu H, Estiu G, Stahelin RV, et al. A molecular mechanism of artemisinin resistance in Plasmodium falciparum malaria. Nature. 2015;520:683-7.

16. Phyo AP, Ashley EA, Anderson TJ, Bozdech Z, Carrara VI, Sriprawat K, et al. Declining efficacy of artemisinin combination therapy against $P$. falciparum malaria on the Thai-Myanmar Border (2003-2013): the role of parasite genetic factors. Clin Infect Dis. 2016;63:784-91.

17. Tilley L, Straimer J, Gnadig NF, Ralph SA, Fidock DA. Artemisinin action and resistance in Plasmodium falciparum. Trends Parasitol. 2016;32:682-96.

18. Amaratunga C, Lim P, Suon S, Sreng S, Mao S, Sopha C, et al. Dihydroartemisinin-piperaquine resistance in Plasmodium falciparum malaria in Cambodia: a multisite prospective cohort study. Lancet Infect Dis. 2016:16:357-65

19. Leang R, Taylor WR, Bouth DM, Song L, Tarning J, Char MC, et al. Evidence of Plasmodium falciparum malaria multidrug resistance to artemisinin and piperaquine in western Cambodia: dihydroartemisinin-piperaquine open-label multicenter clinical assessment. Antimicrob Agents Chemother. 2015:59:4719-26.

20. Eastman RT, Dharia NV, Winzeler EA, Fidock DA. Piperaquine resistance is associated with a copy number variation on chromosome 5 in drug-pressured Plasmodium falciparum parasites. Antimicrob Agents Chemother. 2011:55:3908-16

21. Amato R, Lim P, Miotto O, Amaratunga C, Dek D, Pearson RD, et al. Genetic markers associated with dihydroartemisinin-piperaquine failure in Plasmodium falciparum malaria in Cambodia: a genotype-phenotype association study. Lancet Infect Dis. 2017;17:164-73.

22. Witkowski B, Duru V, Khim N, Ross LS, Saintpierre B, Beghain J, et al. A surrogate marker of piperaquine-resistant Plasmodium falciparum malaria: a phenotype-genotype association study. Lancet Infect Dis. 2017;17:174-83.

23. White NJ. Can new treatment developments combat resistance in malaria? Expert Opin Pharmacother. 2016;17:1303-7.

24. Daniels R, Hamilton EJ, Durfee K, Ndiaye D, Wirth DF, Hartl DL, et al. Methods to increase the sensitivity of high resolution melting single nucleotide polymorphism genotyping in malaria. J Vis Exp. 2015;105:e52839.

25. Daniels R, Volkman SK, Milner DA, Mahesh N, Neafsey DE, Park DJ, et al. A general SNP-based molecular barcode for Plasmodium falciparum identification and tracking. Malar J. 2008;7:223.
26. Miotto O, Amato R, Ashley EA, Maclnnis B, Almagro-Garcia J, Amaratunga $C$, et al. Genetic architecture of artemisinin-resistant Plasmodium falciparum. Nat Genet. 2015;47:226-34.

27. Johnson JD, Dennull RA, Gerena L, Lopez-Sanchez M, Roncal NE, Waters NC. Assessment and continued validation of the malaria SYBR green I-based fluorescence assay for use in malaria drug screening. Antimicrob Agents Chemother. 2007:51:1926-33.

28. Ribacke U, Mok BW, Wirta V, Normark J, Lundeberg J, Kironde F, et al. Genome wide gene amplifications and deletions in Plasmodium falciparum. Mol Biochem Parasitol. 2007;155:33-44.

29. Price RN, Uhlemann AC, Brockman A, McGready R, Ashley E, Phaipun $L$, et al. Mefloquine resistance in Plasmodium falciparum and increased pfmdr1 gene copy number. Lancet. 2004;364:438-47.

30. Lim P, Dek D, Try V, Eastman RT, Chy S, Sreng S, et al. Ex vivo susceptibility of Plasmodium falciparum to antimalarial drugs in western, northern, and eastern Cambodia, 2011-2012: association with molecular markers. Antimicrob Agents Chemother. 2013;57:5277-83.

31. Schwach F, Bushell E, Gomes AR, Anar B, Girling G, Herd C, et al. PlasmoGEM, a database supporting a community resource for large-scale experimental genetics in malaria parasites. Nucleic Acids Res 2015;43: 1176-82. doi:10.1093/nar/gku1143

32. The MalariaGEN Genomic Epidemiology Network Pf3K Project. https:// www.malariagen.net/projects/pf3k. Accessed Mar 2015.

33. Wang Z, Wang Y, Cabrera M, Zhang Y, Gupta B, Wu Y, et al. Artemisinin resistance at the China-Myanmar border and association with mutations in the K13 propeller gene. Antimicrob Agents Chemother. 2015:59:6952-9.

34. Bzik DJ, Li WB, Horii T, Inselburg J. Molecular cloning and sequence analysis of the Plasmodium falciparum dihydrofolate reductase-thymidylate synthase gene. Proc Natl Acad Sci USA. 1987;84:8360-4.

35. Triglia T, Cowman AF. Primary structure and expression of the dihydropteroate synthetase gene of Plasmodium falciparum. Proc Natl Acad Sci USA. 1994;91:7149-53.

36. Leber W, Skippen A, Fivelman QL, Bowyer PW, Cockcroft S, Baker DA. A unique phosphatidylinositol 4-phosphate 5-kinase is activated by ADP-ribosylation factor in Plasmodium falciparum. Int J Parasitol. 2009;39:645-53.

37. Guttery DS, Poulin B, Ramaprasad A, Wall RJ, Ferguson DJ, Brady D, et al. Genome-wide functional analysis of Plasmodium protein phosphatases reveals key regulators of parasite development and differentiation. Cell Host Microbe. 2014;16:128-40.

38. Pandey R, Mohmmed A, Pierrot C, Khalife J, Malhotra P, Gupta D. Genome wide in silico analysis of Plasmodium falciparum phosphatome. BMC Genom. 2014:15:1024

39. Wilkes JM, Doerig C. The protein-phosphatome of the human malaria parasite Plasmodium falciparum. BMC Genom. 2008:9:412.

40. Guttery DS, Poulin B, Ferguson DJ, Szoor B, Wickstead B, Carroll PL, et al. A unique protein phosphatase with kelch-like domains (PPKL) in Plasmodium modulates ookinete differentiation, motility and invasion. PLoS Pathog. 2012;8:e1002948.

41. Zalis MG, Wilson CM, Zhang Y, Wirth DF. Characterization of the pfmdr2 gene for Plasmodium falciparum. Mol Biochem Parasitol. 1993;62:83-92.

42. Rubio JP, Cowman AF. Plasmodium falciparum: the pfmdr2 protein is not overexpressed in chloroquine-resistant isolates of the malaria parasite. Exp Parasitol. 1994;79:137-47.

43. Cheeseman IH, Miller BA, Nair S, Nkhoma S, Tan A, Tan JC, et al. A major genome region underlying artemisinin resistance in malaria. Science. 2012;336:79-82.

44. Takala-Harrison S, Jacob CG, Arze C, Cummings MP, Silva JC, Dondorp $\mathrm{AM}$, et al. Independent emergence of artemisinin resistance mutations among Plasmodium falciparum in Southeast Asia. J Infect Dis. 2015:211:670-9.

45. Chaorattanakawee S, Saunders DL, Sea D, Chanarat N, Yingyuen K, Sundrakes S, et al. Ex vivo drug susceptibility testing and molecular profiling of clinical Plasmodium falciparum isolates from Cambodia from 2008 to 2013 suggest emerging piperaquine resistance. Antimicrob Agents Chemother. 2015;59:4631-43.

46. Chaorattanakawee S, Lanteri CA, Sundrakes S, Yingyuen K, Gosi P, Chanarat N, et al. Attenuation of Plasmodium falciparum in vitro drug resistance phenotype following culture adaptation compared to fresh clinical isolates in Cambodia. Malar J. 2015;14:486. 
47. Duru V, Khim N, Leang R, Kim S, Domergue A, Kloeung N, et al. Plasmodium falciparum dihydroartemisinin-piperaquine failures in Cambodia are associated with mutant K13 parasites presenting high survival rates in novel piperaquine in vitro assays: retrospective and prospective investigations. BMC Med. 2015;13:305
48. Boulle M, Witkowski B, Duru V, Sriprawat K, Nair SK, McDew-White M, et al. Artemisinin-resistant Plasmodium falciparum K13 mutant alleles, Thailand-Myanmar border. Emerg Infect Dis. 2016;22:1503-5.

49. Vaughan AM, Pinapati RS, Cheeseman IH, Camargo N, Fishbaugher M Checkley LA, et al. Plasmodium falciparum genetic crosses in a humanized mouse model. Nat Methods. 2015;12:631-3. 